\title{
Review of: "An integrated pore size distribution measurement method of small angle neutron scattering and mercury intrusion capillary pressure"
}

\author{
qiang zeng $^{1}$ \\ 1 Zhejiang University
}

Potential competing interests: The author(s) declared that no potential competing interests exist.

The authors combined the small-angle neutron scattering (SANS) and mercury intrusion capillary pressure testing (MICP) methods to present integrative investingations on the pore size distribution of the broad sense shale oil reservoir samples of the Permian Lucaogou Formation in the Jimsar Sag, Junggar Basin, China. Basically, any single pore structure probing method cannot provide full pore information due to different reasons. For example, MICP tests can provide broad pore size distribution data, but the samples have to be dried prior to tests, so the microstructure of the samples may be altered due to the building of high capillary pressures on the pore walls in thin pore system during the drying process. This says that the obtained pore size distribution may be different from the original materials. Also, becuase of the high pressures of mercury exterting on the skeletons of materials during MICP tests, serious materials damages may occur (doi:10.3390/ma12142220). For the porous materials with a series of different pore geometries, the 'ink-bottle' effect may also paly a part (https://doi.org/10.1016/j.cemconcomp.2020.103726.).

Therefore, in order to obtain the pore information as accurately as possible, the combination of MICP and other pore structure testing methods can indeed improve the testing accurance. SANS is a non-destructive method to measure the connected pores and closed pores in porous materials with high accurance. In this paper, the combination of MICP and SANS thus can provide more pore information, such as, broader pore size.

In this work, the authors directly plotted the MICP and SANS pore size distributions together, which may be questionable. Because in MICP, it assumes that the pores are cylinders, while in SANS, the pores are assumed to comform to a polydisperse spherical pore network. So the simple cylinder+sphere pores may have problems of compatability. Futher consistent pore models should be developed based on experimental data to demonstrate the 'real' pore system. 